\title{
Propriétés thermodynamiques des solutions liquides bromure de cuivre(I) - bromure de sodium et bromure de cuivre(I) - bromure de potassium à partir des équilibres liquide - solide
}

\author{
A. Wojakowska et E. Krzyzak \\ Laboratoire d'Analyse Thermique, Département de Chimie Minérale, Académie de \\ Médecine, 38 rue Szewska, 50139 Wroclaw, Pologne
}

\begin{abstract}
Partial molar free enthalpies of mixing and activities of the components in molten solutions of the binary systems $\mathrm{CuBr}-\mathrm{NaBr}$ and $\mathrm{CuBr}-\mathrm{KBr}$ have been obtained from the liquidus lines. Both systems indicate negative deviations from ideality, the deviation being more pronounced with increasing size of the alkali metal cation. From the combined results of the integral free enthalpies of mixing and available data on the enthalpies of mixing, the entropies of mixing for the system $\mathrm{CuBr}-\mathrm{NaBr}$ have been calculated and compared with those for the system $\mathrm{CuCl}-\mathrm{NaCl}$. The system $\mathrm{CuBr}-\mathrm{NaBr}$ indicates positive excess entropies, twice as large as in the system $\mathrm{CuCl}-\mathrm{NaCl}$, except in the copper(I) bromide rich solutions (at concentrations above $65 \mathrm{~mol} \%$ ), where they become insignificant. These small excess entropies of mixing may result from the superionic character of the high temperature modifications of copper(I) bromide, having disordered structures.
\end{abstract}

\section{INTRODUCTION}

Les équilibres entre phases pour les systèmes $\mathrm{CuBr}-\mathrm{NaBr}$ et $\mathrm{CuBr}-\mathrm{KBr}$ n'ont pas été décrits en détail jusqu' à présent. Seulement neuf mélanges pour chacun des systèmes ont été considérés pour la construction des diagrammes de phases d'après des données ATD [1]. Le problème d'existence de solutions solides à base des constituants a été laissé ouvert. En revanche, la formation du composé intermédiaire $\mathrm{K}_{2} \mathrm{CuBr}_{3}$ dans le système $\mathrm{CuBr}-\mathrm{KBr}$ a été bien documentée par diffraction des rayons $\mathrm{X}$.

Le présent travail entre dans le cadre d'un programme d'études des systèmes à base d' halogénures de cuivre(I) dans le but de trouver des nouveaux électrolytes solides. Nous avons entrepris l'étude de ces deux systèmes pour déterminer avec précision la courbe de liquidus et calculer les variations de l'enthalpie libre de mélange à l'état liquide en fonction de la composition. Les données obtenues constituent une contribution à la connaissance des propriétés thermodynamiques des solutions liquides binaires et, par consequent, peuvent servir à étudier les propriétes thermodynamiques des systèmes ternaires et leur aptitude à la formation de phases intermédiaires. 


\section{PARTIE EXPÉRIMENTALE}

Les températures de liquidus des systèmes $\mathrm{CuBr}-\mathrm{NaBr}$ et $\mathrm{CuBr}-\mathrm{KBr}$ sont déterminées par calorimetrie à balayage, à l'aide d'un appareil DSC 25 Mettler Toledo. Les détails de la méthode ont été décrits précédemment [2]. Les mélanges à étudier (37 échantillons pour le système $\mathrm{CuBr}-\mathrm{NaBr}$ et 42 échantillons pour le système $\mathrm{CuBr}-\mathrm{KBr}$ ) sont obtenus par synthèse directe dans des cellules en quartz scellées sous vide, en utilisant des sels de haute pureté. Les cellules sont ensuite utilisées dans des expériences de DSC réalisées avec des vitesses de chauffe ou de refroidissement comprises entre 1 et $10 \mathrm{~K} \min ^{-1}$ dans le domaine de température 373-1023 K.

\section{RÉSULTATS}

Le bromure de cuivre(I) montre trois polymorphes sous pression normale [3]. La modification $\gamma$-CuBr, stable à la température ambiante, se transforme à $657 \mathrm{~K}$ en $\beta$-CuBr. Celui-ci se transforme à $741 \mathrm{~K}$ en $\alpha-\mathrm{CuBr}$ qui fond à $759 \mathrm{~K}$.

Le diagramme d'équilibre de phases du système $\mathrm{CuBr}-\mathrm{NaBr}$ présente une eutexie. Le pic correspondant est observé sur les courbes DSC à $603 \mathrm{~K}$ dans tout le domaine de composition. Le diagramme de Tamman indique la composition du point eutectique à $\mathrm{x}_{\mathrm{CuBr}}=0.71$ ainsi que l'absence de solutions solides à base de $\mathrm{NaBr}$ et de $\gamma-\mathrm{CuBr}$.

Le diagramme d'équilibre de phases du système $\mathrm{CuBr}-\mathrm{KBr}$ revèle la formation du composé $\mathrm{K}_{2} \mathrm{CuBr}_{3}$ à fusion non congruente à $507 \mathrm{~K}$. Le pic correspondant est observé sur les courbes DSC à partir des compositions très proches du $\mathrm{KBr}$ pur jusqu' au point péritectique à $\mathrm{x}_{\mathrm{CuBr}}=0.56$. Le mélange eutectique a une composition $\mathrm{x}_{\mathrm{CuBr}}=0.62$ et fond à $452 \mathrm{~K}$. L'invariance eutectique à $452 \mathrm{~K}$ est observée sur les courbes DSC entre $\mathrm{x}_{\mathrm{CuBr}}=0.356$ et $\mathrm{x}_{\mathrm{CuBr}}=0.995$. Les diagrammes de Tamman correspondants confirment la composition du composé et ceux du point péritectique et du point eutectique ainsi que l'absence de solutions solides à base de $\mathrm{KBr}$ et de $\gamma$-CuBr .

Les deux diagrammes d'équilibre comportent des lignes invariantes, relatives aux transformations polymorphiques du bromure de cuivre(I) : $\gamma-\mathrm{CuBr} \Leftrightarrow \beta-\mathrm{CuBr}$ et $\beta-\mathrm{CuBr} \Leftrightarrow \alpha-\mathrm{CuBr}$. Elles sont observées dans les domaines de composition entre le liquidus et $\mathrm{CuBr}$ pur.

A partir des diagrammes d'équilibre de phases décrits ci-dessus, il a été possible de calculer les enthalpies libres molaires partielles de mélange des composants $\mathbf{G}_{\mathbf{i}}$, dans le système $\mathrm{CuBr}-\mathrm{NaBr}$ pour tout le domaine de composition (en utilisant l'équation de Gibbs-Duhem), tandis que dans le système $\mathrm{CuBr}-\mathrm{KBr}$ cela a été limité aux enthalpies libres molaires partielles de mélange de $\mathrm{KBr}$ dans le domaine de fraction molaire $\mathrm{CuBr}$ 0-0.56 et celles de $\mathrm{CuBr}$ dans le domaine de fraction molaire $\mathrm{CuBr}$ 0.62-1 (Figure1). Pour les mêmes domaines de composition nous avons aussi déterminé les enthalpies libres molaires partielles d'excès des composants $\mathbf{G}_{\mathbf{i}}{ }^{\mathbf{E}}$ (Figure 1), leurs activités $\mathbf{a}_{\mathbf{i}}$ et coefficients d'activité $\mathbf{f}_{\mathbf{i}}$ dans les mélanges fondus (Figure 2). On a noté que dans le système $\mathrm{CuBr}-\mathrm{NaBr}$ les fonctions $\mathbf{G}_{\mathbf{i}} \mathbf{E}$ et $\mathbf{f}_{\mathbf{i}}$ se stabilisent pour des solutions très diluées, s'approchant des valeurs $\mathbf{G}_{\mathbf{i}}^{\mathbf{E}}=-2.5 \mathrm{~kJ} \mathrm{~mol}^{-1}$ et $\mathbf{f}_{\mathbf{i}}=0.67$ pour $\mathrm{NaBr}$ et $\mathbf{G}_{\mathbf{i}}^{\mathbf{E}}=-7.9 \mathrm{~kJ} \mathrm{~mol}^{-1}$ et $\mathbf{f}_{\mathbf{i}}=0.39$ pour $\mathrm{CuBr}$. 

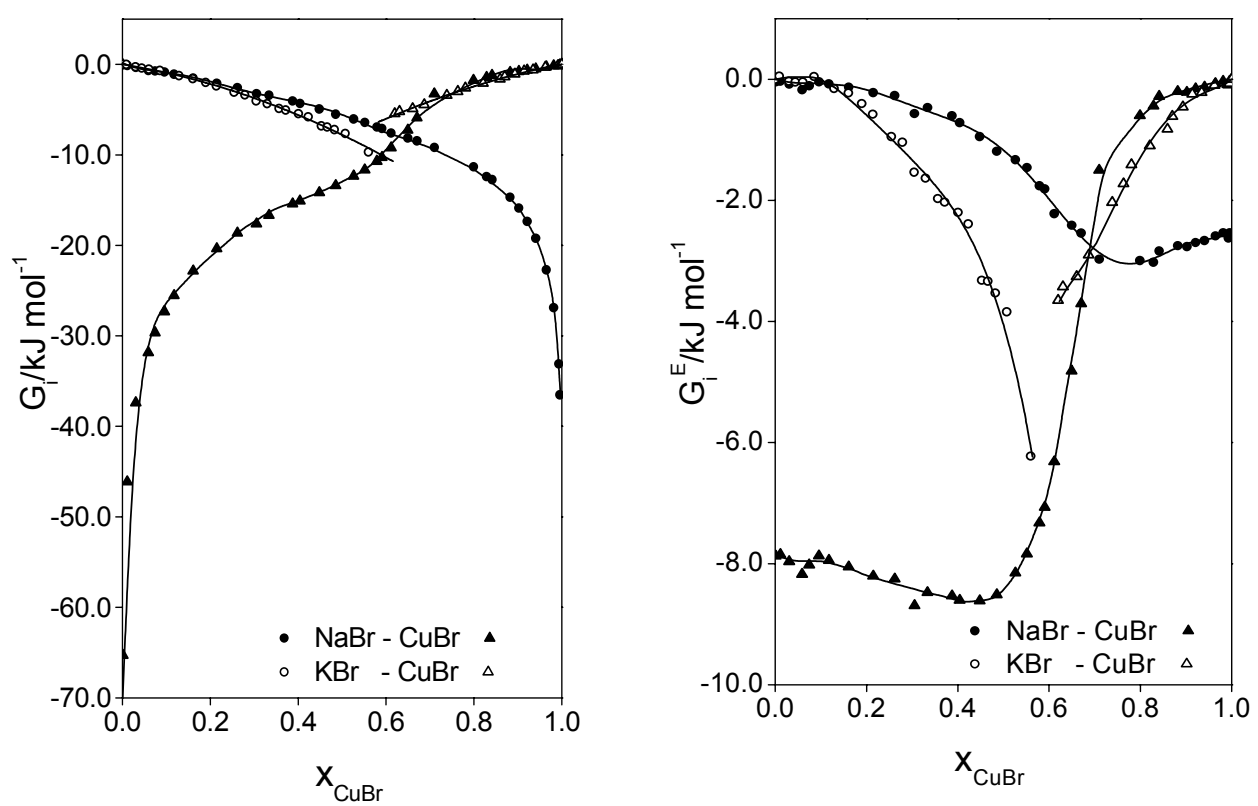

Figure 1. Enthalpies libres molaires partielles de mélange des composants $\mathbf{G}_{\mathbf{i}}$ et enthalpies libres molaires partielles d'excès des composants $\mathbf{G}_{\mathbf{i}} \mathbf{E}$ pour le système $\mathrm{CuBr}-\mathrm{NaBr}$ et pour le système $\mathrm{CuBr}-\mathrm{KBr}$.
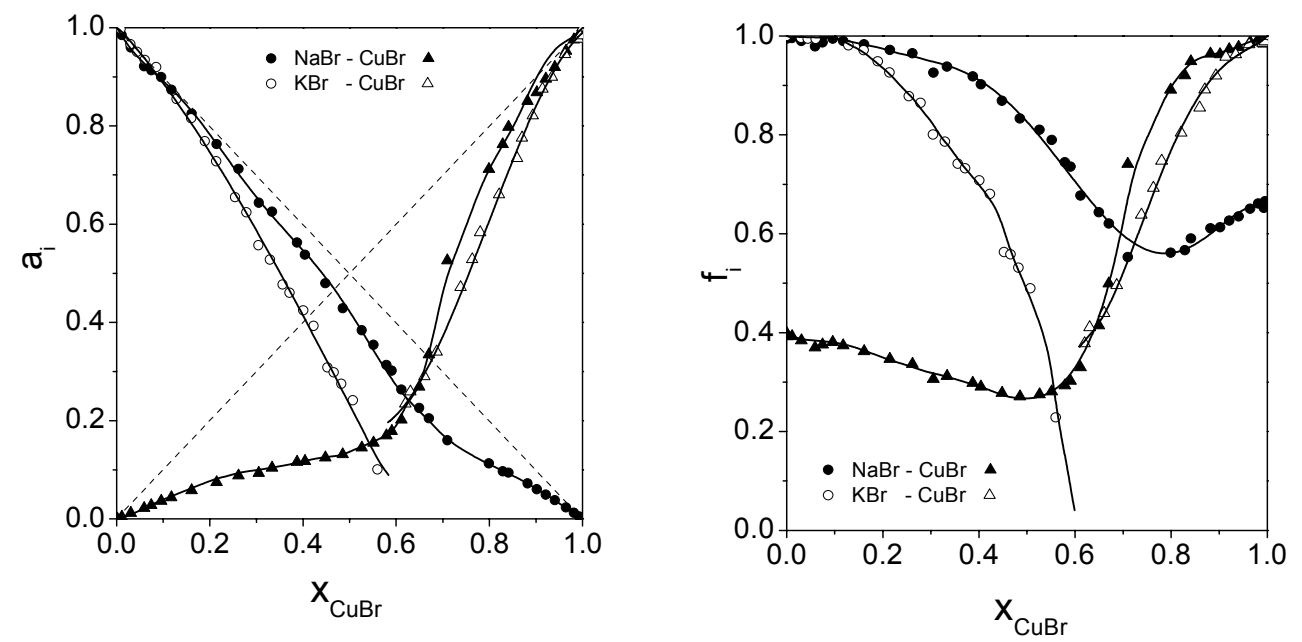

Figure 2. Activités $\mathbf{a}_{\mathbf{i}}$ des composants et coefficients d'activité $\mathbf{f}_{\mathbf{i}}$ dans les mélanges fondus $\mathrm{CuBr}-\mathrm{NaBr}$ et $\mathrm{CuBr}-\mathrm{KBr}$.

Le calcul des enthalpies libres molaires partielles de mélange du composant, qui cristallise à la température $\mathrm{T}$ en équilibre avec la solution, demande la connaissance des valeurs de l'enthalpie de fusion $\left(\Delta_{\text {fus }} \mathrm{H}\right)$ et celle de la chaleur spécifique de ce composant à l'état liquide $\left(\mathrm{C}_{\mathrm{p}}{ }^{1}\right)$ et à l'état solide $\left(\mathrm{C}_{\mathrm{p}} \mathrm{s}\right)$. Si le composant présente des transitions polymorphiques et dans les domaines des températures respectifs peut cristalliser sous plusieurs formes: $\alpha, \beta, \gamma \ldots$, le calcul des enthalpies libres molaires partielles de mélange demande la connaissance des valeurs des enthalpies des transitions polymorphiques: $\Delta_{\mathrm{tr}} \mathrm{H}_{\alpha / \beta}, \Delta_{\mathrm{tr}} \mathrm{H}_{\beta / \gamma}, \ldots$ et des chaleurs spécifiques des variétés successives [4]. 
Les valeurs utilisées dans ce travail pour $\mathrm{CuBr}$ [5] et les bromures alcalins $\mathrm{NaBr}$ et $\mathrm{KBr}$ [6] sont indiquées dans le tableau 1.

Tableau 1. Valeurs de $\Delta_{\text {fus }} \mathrm{H} / \mathrm{kJ} \mathrm{mol}^{-1}, \Delta_{\mathrm{tr}} \mathrm{H} / \mathrm{kJ} \mathrm{mol}^{-1}$ et de $\mathrm{C}_{\mathrm{p}}=\mathrm{A}+\mathrm{B} \mathrm{T}+\mathrm{C} \mathrm{T}^{-2} / \mathrm{J} \mathrm{K}^{-1} \mathrm{~mol}^{-1}$.

\begin{tabular}{|c|c|c|c|c|c|c|}
\hline & enthalpie de transition & Phase & A & $\mathrm{B} ! 0^{3}$ & $\mathrm{C} 10^{-5}$ & Ref. \\
\hline $\mathrm{NaBr}$ & $\Delta_{\text {fus }} \mathrm{H}=26.100$ & $\begin{array}{l}\text { liquide } \\
\text { solide }\end{array}$ & $\begin{array}{l}62.34 \\
47.91\end{array}$ & 13.31 & & 6 \\
\hline $\mathrm{KBr}$ & $\Delta_{\text {fus }} \mathrm{H}=25.500$ & $\begin{array}{l}\text { liquide } \\
\text { solide }\end{array}$ & $\begin{array}{l}69.87 \\
69.16\end{array}$ & -45.56 & -6.49 & 6 \\
\hline $\mathrm{CuBr}$ & $\begin{array}{l}\Delta_{\text {fus }} \mathrm{H}=5.104 \\
\Delta_{\operatorname{tr}} \mathrm{H}_{\alpha / \beta}=2.134 \\
\Delta_{\mathrm{tr}} \mathrm{H}_{\beta / \gamma}=4.602\end{array}$ & \begin{tabular}{|l} 
liquide \\
solide $\alpha$ \\
solide $\beta$ \\
solide $\gamma$
\end{tabular} & \begin{tabular}{|l|}
45.953 \\
58.158 \\
73.224 \\
-51.999
\end{tabular} & $\begin{array}{l}35.7 \\
206.4\end{array}$ & $\begin{array}{l}84.835 \\
40.17\end{array}$ & 5 \\
\hline
\end{tabular}

Pour le système $\mathrm{CuBr}-\mathrm{NaBr}$ nous avons été en mesure de calculer les enthalpies libres molaires intégrales de mélange $\mathbf{G}^{\mathbf{M}}$ et les enthalpies libres molaires intégrales d'excès $\mathbf{G}^{\mathbf{E}}$ (Figure 3). A partir de nos résultats sur les enthalpies libres molaires partielles d'excès de $\mathrm{CuBr}$ et de $\mathrm{NaBr}$ dans le système $\mathrm{CuBr}-\mathrm{NaBr}$, nous avons calculé les enthalpies libres molaires d'excès de mélange dans toute l'étendue du domaine de composition. En utilisant ces valeurs et celles des enthalpies de mélange, determinées par Dantzer [7], nous avons pu évaluer les valeurs des entropies molaires intégrales de mélange $\mathbf{S}^{\mathbf{M}}$ et les entropies molaires intégrales d'excès $\mathbf{S}^{\mathbf{E}}$ (Figure 3) dans le système $\mathrm{CuBr}-\mathrm{NaBr}$.

\section{DISCUSSION}

L'analyse thermodynamique revèle que les écarts d'enthalpie libre que présentent les mélanges fondus étudiés par rapport à un comportement idéal de la solution sont négatifs et généralement plus importants dans le système $\mathrm{CuBr}-\mathrm{KBr}$ que dans le système $\mathrm{CuBr}-\mathrm{NaBr}$ (Figure 1, Figure 2).
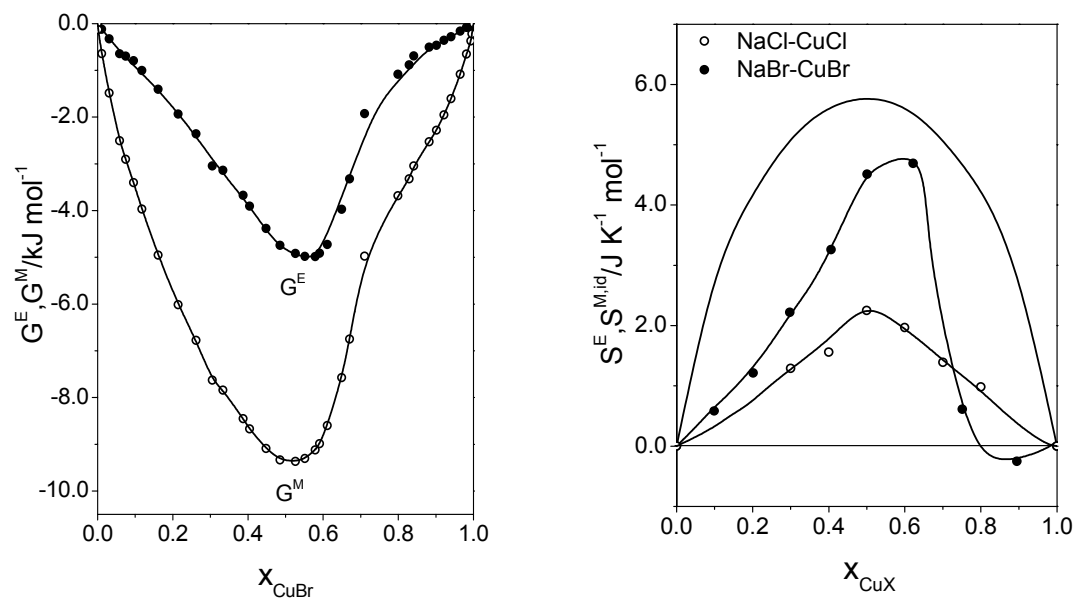

Figure 3. Enthalpies libres molaires intégrales de mélange $\mathbf{G}^{\mathbf{M}}$ et enthalpies libres molaires intégrales d'excès $\mathbf{G}^{\mathbf{E}}$ des mélanges $\mathrm{CuBr}-\mathrm{NaBr}$ [ce travail]. Entropies molaires intégrales d'excès $\mathbf{S}^{\mathbf{E}}$ des mélanges $\mathrm{CuBr}-\mathrm{NaBr}$ et $\mathrm{CuCl}-\mathrm{NaCl}$ (calculées d'après ce travail, [7] et [10]) et l'entropie intégrale idéale de mélange $\mathbf{S}^{\mathbf{M}} \mathbf{i d}$ ). 

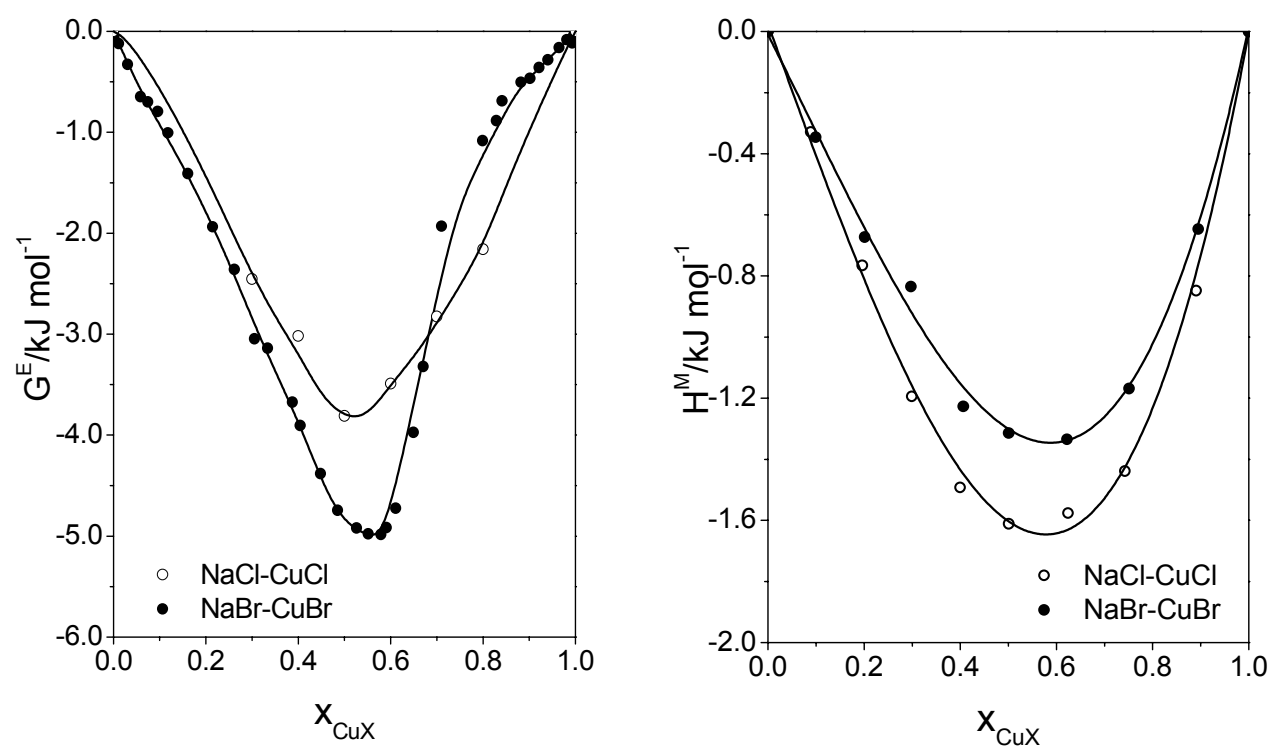

Figure 4. Enthalpies libres molaires intégrales d'excès $\mathbf{G}^{\mathbf{E}}$ des mélanges $\mathrm{CuCl}-\mathrm{NaCl}[10]$ et $\mathrm{CuBr}-\mathrm{NaBr}$ [ce travail]. Enthalpies molaires intégrales de mélange $\mathbf{H}^{\mathbf{M}}$ dans les systèmes $\mathrm{CuCl}-\mathrm{NaCl}$ [7] et $\mathrm{CuBr}-\mathrm{NaBr}$ [7].

Une pareille variation de l'enthalpie libre partielle d'excès vers des valeurs plus négatives a été remarquée pour les mélanges $\mathrm{CuCl}-\mathrm{NaCl}$ et $\mathrm{CuCl}-\mathrm{KCl}$ [8]. Cela suggère la présence d'entités complexes du type $\mathrm{CuX}_{\mathrm{n}}{ }^{(\mathrm{n}-1)-}(\mathrm{X}=\mathrm{Cl}, \mathrm{Br})$ dont la stabilité dans le mélange s'accroît généralement avec la taille du cation alcalin. L'étude expérimentale des systèmes liquides $\mathrm{CuCl}-\mathrm{MCl}(\mathrm{M}=\mathrm{Li}, \mathrm{Na}, \mathrm{K}, \mathrm{Rb}, \mathrm{Cs})$ par la mesure des pressions de vapeurs [9], forces électromotrices [10,11], enthalpies de mélange [7,12], coefficients de diffusion [13] et surtout par spectroscopie Raman [14,15] confirme la possibilité d'associations de ce type.

Les données pour les systèmes des bromures correspondants ne sont pas si nombreuses. Nous notons, néanmoins, que les déviations de la solution idéale $\left(\mathrm{G}^{\mathrm{E}}, \mathrm{H}^{\mathrm{M}}\right)$ observés dans les systèmes à base de $\mathrm{CuBr}$ sont de même ordre que celles observés dans les systèmes à base de $\mathrm{CuCl}$. Ceci est montré dans la Figure 4 où nous présentons les enthalpies libres d'excès de mélanges [ce travail, 10] et les enthalpies de mélange [7] des solutions liquides $\mathrm{CuBr}-\mathrm{NaBr}$ en comparaison avec $\mathrm{CuCl}-\mathrm{NaCl}$. En ce qui concerne l'entropie, les déviations maximales dans le système $\mathrm{CuBr}-\mathrm{NaBr}$ (Figure 3) sont deux fois plus grandes que celles dans le système $\mathrm{CuCl}-\mathrm{NaCl}$. Il paraît donc que des écarts de l'idéalité dans les mélanges $\mathrm{CuBr}-\mathrm{NaBr}$ sont pour la plupart de type entropique tandis que ceux dans les mélanges $\mathrm{CuCl}-\mathrm{NaCl}$ sont de type enthalpique.

D'autre part, dans le domaine de concentration où les mélanges liquides $\mathrm{CuBr}-\mathrm{NaBr}$ sont en équilibre avec $\mathrm{CuBr}$, on note que l'entropie de mélange est presque idéale, ce qui n'est pas observé pour les solutions riches en $\mathrm{CuCl}$ dans le système $\mathrm{CuCl}-\mathrm{NaCl}$ (Figure 3). Il faut remarquer que les modifications $\alpha-\mathrm{CuBr}$ et $\beta$ - $\mathrm{CuBr}$ sont considerées comme des phases superioniques. Elles ont des structures particulières permettant l'existence d'une concentration élevée de défauts de réseau cristallin. Ceci évoque déjà les propriétés de l'état liquide et, par consequant, implique un faible changement de l'entropie au cours de la formation du mélange fondu. 


\section{Références}

[1] Biefeld R. M., Mater. Res. Bull. 10 (1975) 1151-1156

[2] Wojakowska A., Górniak A. et Wojakowski A., J. Phys IV France 113 (2004) 159-162

[3] Rapoport E. et Pistorius C. W. F. T., Phys. Rev. 172 (1968) 838-847

[4] Wojakowska A., J. Chim. Phys. Physicochim. Biol., 90 (1993) 561-578

[5] Ferrante M. J. et Brown R. R., U. S. Bur. Mines, Rep. Invest. 8917 (1984)

[6] Kubaschewski O., Alcock C. B. et Spencer P. J., Materials Thermochemistry, Pergamon Press, Oxford 1993

[7] Dantzer P., J. Phys. Chem., 85 (1981) 724-727

[8] Mirabel P. et Guion J., Compt. Rend. Acad. Sci., Ser. C 269 (1969) 516-519

[9] Mielcarski M., Z. Anorg. Allg. Chem. 386 (1971) 345-352

[10] Mirabel P., Dantzer P. et Guion J., J. Chim. Phys. Physicochim. Biol. 69 (1972) 268-276

[11] Giazitzoglou Z., Electrochim. Acta 28 (1983) 491- 494

[12] Dantzer P. et Kleppa O. J., Inorg. Chem., 12 (1973) 2699-2701

[13] Poignet J. C. et Barbier M. J., Chem. Zvesti, 32 (1978) 787-790

[14] Gilbert B., Fung K. W., Mamantov G. et Begun G. M., J. Inorg. Nucl. Chem. 37 (1975) 921-927

[15] Christensen E., Berg R. W. et Von Barner J. H., Polyhedron 8 (1989) 325-332 